\title{
Cervical dissecting extravasation of oxaliplatin: A case report
}

\author{
JORGE HERNANDO ${ }^{1}$, JUDIT RIERA-ARNAU ${ }^{2}$, MARIA ROCA ${ }^{1}$, \\ ALEJANDRO GARCIA $^{1}$ and JAUME CAPDEVILA ${ }^{1}$

\begin{abstract}
${ }^{1}$ Gastrointestinal and Endocrine Tumors Unit, Department of Medical Oncology, Vall d'Hebron Institute of Oncology;
${ }^{2}$ Clinical Pharmacology Service, Department of Pharmacovigilance and Pharmacoepidemiology,

Vall d'Hebron University Hospital, Autonomous University of Barcelona, 08035 Barcelona, Spain
\end{abstract}

Received July 23, 2021; Accepted November 9, 2021

DOI: $10.3892 / \mathrm{mco} .2022 .2493$

\begin{abstract}
Oxaliplatin is a chemotherapy drug considered to be an irritant and vesicant. Peripheral extravasation could happen following oxaliplatin chemotherapy administration, but mediastinal or cervical events are rare. The present study focused on the case of a 64-year-old female patient with KRAS-mutated colorectal adenocarcinoma. The patient was prescribed folinic acid, fluorouracil and oxaliplatin chemotherapy via a subcutaneous pump or port-a-cath device, which was inserted into the right subclavian vein. The patient reported a sudden throbbing pain in the chest wall and anterior cervical region. After performing a computed tomography scan, anterior cervical collection and jugular-subclavian venous confluence at the distal end was observed at the venous access site of the subcutaneous port-a-cath device, which extended cranially, dissected cervical planes and forming a hydro-aerial collection in the submaxillary region. Subsequently, the port-a-cath device was removed and a warm dry compress was applied. After 2 weeks, the patient had fully recovered without any sequelae at the cervical level. To the best of the authors' knowledge, this is the first case of cervical extravasation of oxaliplatin reported in the literature to date and will help to manage similar situations.
\end{abstract}

\section{Introduction}

Oxaliplatin is a cytostatic antineoplastic drug that belongs to a novel class of platinum compounds, in which the platinum ion forms a complex with 1,2-diaminocyclohexane and an oxalate group (1). Previous studies on the mechanism of action of oxaliplatin, although not fully elucidated, have reported that the hydrated derivatives formed due to the biotransformation of oxaliplatin interact with DNA. These molecules form intra- and inter-stranded bridges with DNA and, therefore, interrupt DNA

Correspondence to: Dr Judit Riera-Arnau, Clinical Pharmacology Service, Department of Pharmacovigilance and Pharmacoepidemiology, Vall d'Hebron University Hospital, Autonomous University of Barcelona, 119-129 Vall d'Hebron Avenue, 08035 Barcelona, Spain

E-mail: j.riera.ar@gmail.com

Key words: oxaliplatin, extravasation synthesis, which results in the cytotoxic and antitumor activity of oxaliplatin $(1,2)$. Cisplatin, carboplatin and oxaliplatin are composed of double-charged platinum ions surrounded by four ligands. The amine ligands are situated on the left, which form strong interactions with the platinum ion, whereas the chloride ligands or carboxylate compounds are situated on the right, which form leaving groups allowing the platinum ion to form bonds with DNA bases $(3,4)$.

Oxaliplatin was considered to be a non-vesicant until the 2000s when tissue necrosis from extravasation was described (5). However, in certain reviews oxaliplatin is classified as an irritant (6). At present oxaliplatin is considered to have both irritant and vesicant properties $(6,7)$.

In cancer therapy, extravasation refers to the inadvertent infiltration of chemotherapy into the subcutaneous or subdermal tissues surrounding the intravenous or intra-arterial administration site (6). When extravasation occurs the degree of damage is dependent on the agent, the drug concentration, dose and volume, its $\mathrm{pH}$ (7.35-7.40) and osmolarity distance from normality $(281-282 \mathrm{mOsm} / \mathrm{l})$, as well as the location of the extravasation event and the duration of cell exposure to the drug (8). Patient-dependent risk factors of chemotherapy extravasation include small and/or fragile veins, lymphedema, obesity, impaired level of consciousness and numerous previous venipunctures. Iatrogenic factors, which can contribute to extravasation, include lack of proper nursing staff training, selection of the wrong cannula size, suboptimal location, accidental puncturing of the vein or movement of the cannula due to patient movement or insecure fixing $(9,10)$. The extravasation severity and the potential sequelae highlight the fact that it is important to distinguish extravasation from other local reactions to chemotherapy. It is also crucial that the associated risk of the cannulation procedure is reduced and that medical staff are aware of the signs and symptoms of extravasation and are familiar with its management.

\section{Case report}

A 64-year-old female patient presented with a 4-month history of anorectal pain, tenesmus, asthenia and weakness. The patient's medical history included arterial hypertension and diabetes mellitus. A colonoscopy revealed an ulcerated mass within $10 \mathrm{~cm}$ of the anal verge and the histological report determined this to be a colorectal adenocarcinoma. Molecular 
analysis of the mass determined the tumor to have microsatellite stability and a KRAS mutation. A computed tomography (CT) scan of the chest, abdomen and pelvis revealed a voluminous rectal mass with vaginal fistulation and vesical infiltration, as well as numerous mesenteric and retroperitoneal adenopathies. The patient was diagnosed with a rectal adenocarcinoma with unresectable metastatic adenopathies. A folinic acid, fluorouracil and oxaliplatin (FOLFOX-4) chemotherapy regimen was selected as the most suitable approach. Treatment was initiated using a subcutaneous pump for ambulatory administration, with venous access to the right subclavian vein via a port-a-cath device. The port-a-cath was inserted in 2017, using ultrasound to guide the catheter along the subclavian vein. Following its insertion, an X-ray was performed to check the central line position was correct and to scan for any initial complications.

Following three cycles of chemotherapy, the patient exhibited no signs of significant toxicity. However, during the fourth cycle of FOLFOX-4 treatment, the patient reported a sudden throbbing pain in the chest wall and anterior cervical region, with the appearance of a soft edema that coincided with these painful areas. As extravasation of oxaliplatin was suspected, the infusion was interrupted, the content of the port-a-cath device was aspirated and local dry heat was applied. A bolus of intravenous dexamethasone (12 mg) was administered and the patient was referred to the emergency department for further monitoring and treatment.

An urgent blood test revealed the elevation of C-reactive protein; however, this was the only abnormality detected in the patient's blood. A CT scan identified an anterior cervical collection and jugular-subclavian venous confluence at the distal end, with presence of bubbles close to the venous access site of the subcutaneous port-a-cath, which extended cranially dissecting laterocervical planes and formed a hydro-aerial collection located in the submaxillary region of 8x15x25 mm. Furthermore, subcutaneous inflammation was identified in the upper third and anterior thorax, ascending through laterocervical planes to the homolateral submaxillary region (Figs. 1 and 2).

The patient was admitted to the hospital for monitoring and continued steroid (dexamethasone $4 \mathrm{mg}$ iv/8 h) and analgesic treatment. The patient was not treated with antibiotics, as she remained afebrile and exhibited no signs of infection. Edema and pain had improved at $12 \mathrm{~h}$ following admission and erythema and local induration were present in the thoracic and cervical area where extravasation had occurred. The port-a-cath device was removed without complications. The device did not show signs of damage, holes or any leakage points.

A CT scan was performed 10 days after admission and demonstrated resolution of the cervical hydro-aerial collection and a significant improvement in inflammation, which was non-existent in the cervical area but persisted in the anterior thoracic region (Fig. 3). The patient did not present with any sequelae at the cervical level 2 weeks following discharge from hospital. In the thoracic region, a scar-like induration was observed in the area of greatest exposure to the extravasation event; however, this was not painful. Skin integrity was maintained throughout the process. Due to this extravasation event, the patient refused to continue chemotherapy treatment and succumbed to disease progression after 12 months.

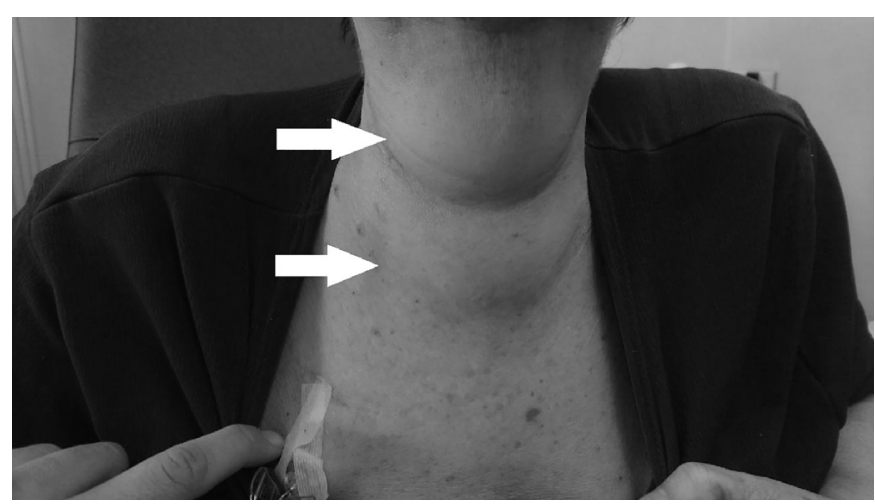

Figure 1. Upper thoracic and cervical swelling (arrowed) during oxaliplatin infusion.

\section{Discussion}

Extravasation of chemotherapy agents administered via a central venous access device (CVAD) is a rare complication. Its prevalence can range from $0.1-6 \%$ when chemotherapy is administered via a peripheral intravenous access point and from $0.26-4.7 \%$ when administered via a CVAD $(11,12)$. In the case of CVAD, when extravasation occurs the drug solution may accumulate in the mediastinum, pleura or in a subcutaneous area of the chest or neck. The most frequent symptom of central line extravasation is acute thoracic pain. Diagnosis of this condition should be based on clinical presentation and further confirmed by imaging, such as a thoracic CT scan. Current data on the management and evolution of chemotherapy extravasation of CVAD are currently based on previous case reports $(7,13)$. As demonstrated in the present study, the management of oxaliplatin extravasation should include discontinuing the infusion and aspirating as much of the chemotherapeutic agent as possible via the CVAD. If the extravasated agent is an anthracycline, dexrazoxane administration may be considered as an antidote. However, in previously reported cases, conventional therapy was the preferred approach, whereby surgical procedures, with the objective of draining the remaining solution, may also be considered $(14,15)$. Antibiotics, endovenous corticosteroids, analgesia and other treatments leading to the control of the symptoms derived from the mediastinitis or pleuritis secondary to extravasation, should also be administered as appropriate $(7,13)$.

Common symptoms related to extravasation include tingling, burning, discomfort/pain, swelling and redness at the injection site $(16,17)$. Late symptoms may include blistering, necrosis and ulceration. Signs that indicate extravasation may occur are the absence of blood return, resistance on the plunger of the syringe during delivery of a bolus drug, or an interruption to the free flow of an infusion (7). Supportive and non-specific approaches have been described in managing cytostatic drug extravasation, as there are currently no direct antidotes (8). Prolonged peripheral line infusions of vesicants are associated with an increased risk of extravasation (8). Therefore, vesicants should not be administered as prolonged unsupervised infusions via peripheral veins, as they are more likely to cause complications, including tissue necrosis. If this occurs, the infusion should be stopped immediately. When several rounds of treatment are needed and there are pre-existing extravasation risk factors, it is recommended that the drug is administered via a central line $(8,9,16)$. 

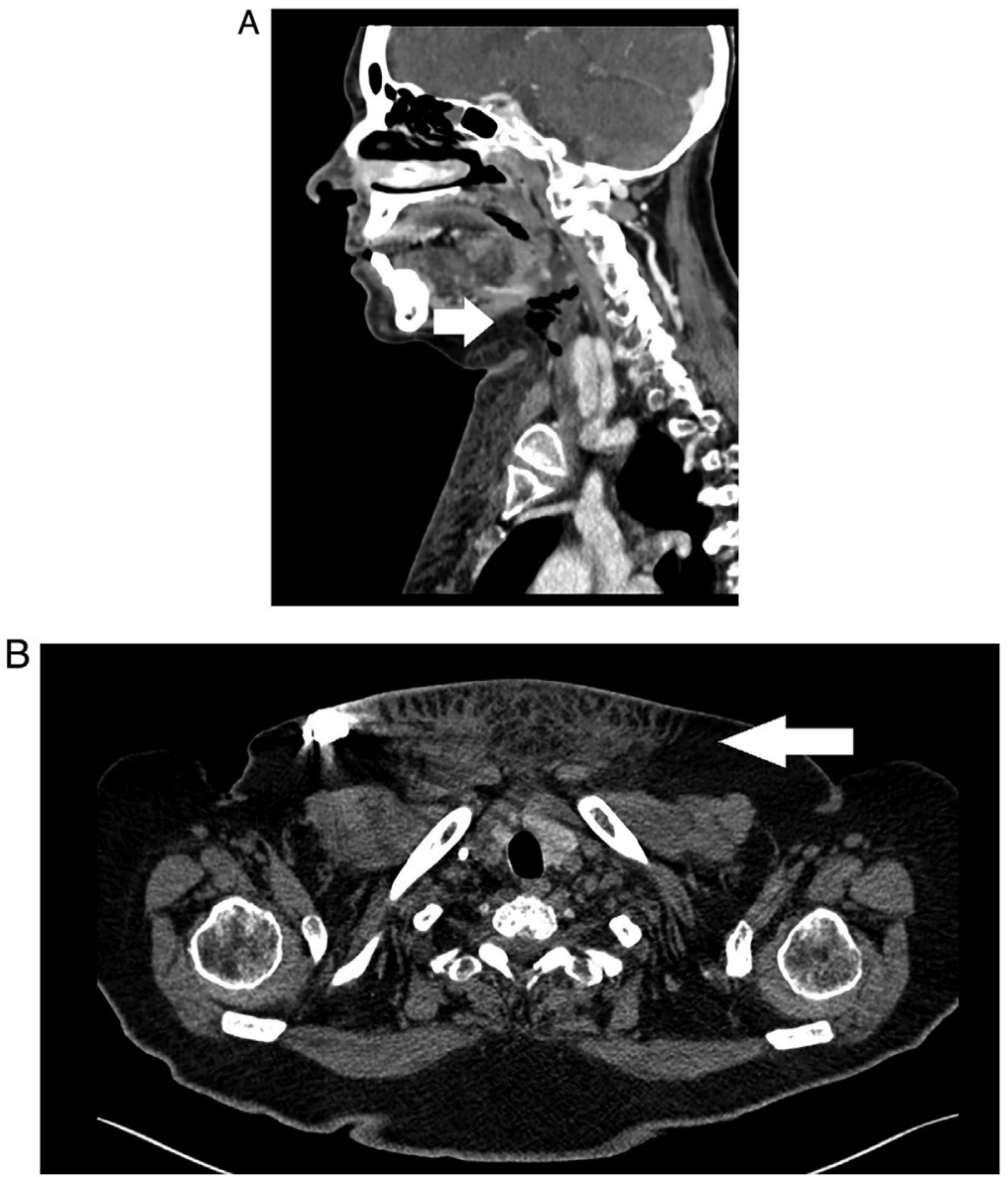

Figure 2. Computed tomography scan images of extravasation. (A) Air bubbles (arrowed) extended cranially, dissecting laterocervical planes. (B) Subcutaneous inflammation (arrowed) was observed in the upper thoracic wall.

Extravasation via a central line is most commonly caused by a dislodged needle in the port (15). It is therefore important that the proper positioning of the port is assessed using imaging following the procedure. Previous case studies have also described this procedure in patients with peripherally inserted central catheters (11). Oncology nurses must follow institutional protocols for the assessment of central venous access if extravasation occurs while using a CVAD (14). Furthermore, when managing extravasation, it has been reported that hypertonic solutions can further increase tissue injury and lead to tissue necrosis (9), whereas cold treatment can aggravate neuropathy. A warm compress may increase drug removal by local vasodilation, which may help avoid peripheral neuropathy (18); however, it may potentially increase cellular uptake and, therefore, injury. Further research into this area is needed $(4,19)$.

A study identifying five oxaliplatin extravasation cases, including a case involving high doses of oxaliplatin $(>40 \mathrm{mg}$ ), where local cooling was applied and induration and localized pain were exhibited by several patients, was previously published (20). However, this treatment strategy is not recommended, as the application of localized cold treatment with a cytostatic compound may increase the risk of peripheral neuropathy. Therefore, the application of local heat is currently recommended, although, to the best of the authors' knowledge, there are no studies describing this treatment. It is a common practice to apply heat, for

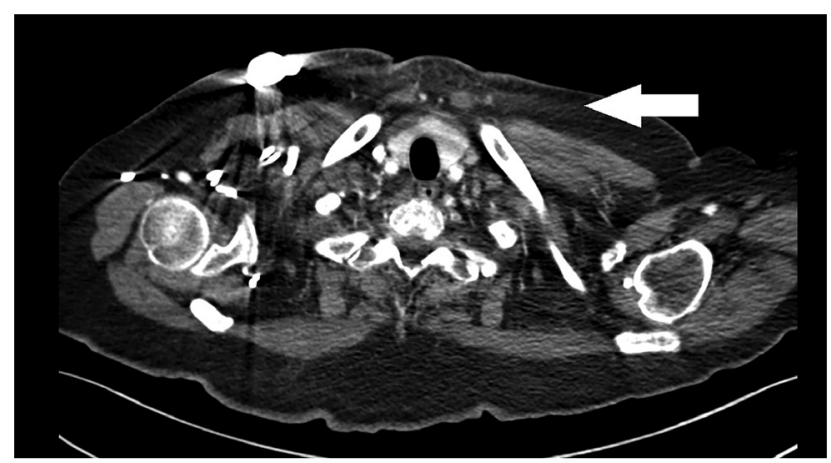

Figure 3. Computed tomography scan images on day 10 following admission. Imaging findings indicated resolution of the cervical hydro-aerial collection (arrowed).

$30 \mathrm{~min}$, or for $15 \mathrm{~min}$ every $6 \mathrm{~h}$ over 2 days $(4,5,11,20-22)$. Treatment recommendations rarely come from controlled clinical trials, but are often based on animal models, case studies or small uncontrolled studies, due to the following: Ethics, the rarity of the event, the lack of clear definition in the efficacy of treatments (such as surgical intervention), impairment of mobility and aesthetic sequelae and poorly studied anecdotal treatment strategies (including sulphadiazine and corticoids) $(12,18)$.

In summary, there are currently few guidelines on how to manage oxaliplatin extravasation. In most cases, a warm, dry 
compress is applied, in contrast to other platins, which require wet dressings.

The cervical extravasation of other specific drugs has also previously been described in detail $(17,18)$, such as in a patient treated with bevacizumab for colorectal cancer. In that case, extravasation occurred during chemotherapy infusion due to a catheter migration of the port outside of the superior vena cava, causing cervical pain without skin manifestations. Conservative management was proposed. The patient fully recovered from all symptoms within 3 weeks. Physicians should also be aware that, in cases of bevacizumab extravasation, a non-surgical approach may be effective. To the best of the authors' knowledge, there has only been a single case report on tissue damage following paravasal infusion of oxaliplatin. In this previous case report, a 62-year-old male patient with colon cancer received adjuvant chemotherapy and presented with extensive tissue damage following oxaliplatin extravasation in the left antecubital region. Despite the severity of the extravasation event and a prolonged stay in hospital, the patient had almost fully recovered at 8 months, without surgical intervention. The patient had exhibited a high temperature and presented with clinical signs of infection; however, directed treatment using several antibiotics was ineffective. Recovery occurred gradually following extravasation, including lymph drainage and the administration of prednisone (12).

In conclusion, cervical extravasation of oxaliplatin is a unique event that has not previously been reported in the literature, to the best of the authors' knowledge. The increased use of central venous catheters to infuse this drug may lead to similar cases being reported in the future. Although severe tissue inflammation and necrosis have been reported in cases involving soft tissue, the present study suggested that cervical oxaliplatin extravasation can be managed with close observation and symptomatic treatment alone. Individual cases, however, may require a more aggressive approach. It is crucial that roper catheter positioning is confirmed prior to drug administration.

\section{Acknowledgements}

Not applicable.

\section{Funding}

No funding was received.

\section{Availability of data and materials}

The datasets used and/or analyzed during the current study are available from the corresponding author on reasonable request.

\section{Authors' contributions}

$\mathrm{JH}$ and JRA contributed to the literature review, writing the manuscript, analysis of clinical information and case discussion. MR, AG and JC analyzed patient data and advised on patient treatment. JH and JRA confirm the authenticity of all the raw data. All the authors have read and approved the final version of the manuscript.

\section{Ethics approval and consent to participate}

Patient consent for publication was approved by ethics approval from Vall d'Hebron University Hospital (Spain).

\section{Patient consent for publication}

Written informed consent was obtained from the family delegate of the patient for publication of the clinical data and any accompanying images.

\section{Competing interests}

The authors declare that they have no competing interests.

\section{References}

1. Yuan X, Zhang W, He Y, Yuan J, Song D, Chen H, Qin W, Qian X, Yu H and Guo Z: Proteomic analysis of cisplatin- and oxaliplatin-induced phosphorylation in proteins bound to Pt-DNA adducts. Metallomics 12: 1834-1840, 2020.

2. Spanish Agency for Medicines and Health Products (AEMPS): Technical datasheet of oxaliplatin. AEMPS, Madrid, 2021. https://cima.aemps.es/cima/publico.html. Accessed January 22, 2021.

3. Goodsell DS: The molecular perspective: Cisplatin. Oncologist 11: 316-317, 2006.

4. Bahadori F and Demiray M: Management of extravasation of oxaliplatin by mimicking its biotransformation. Clin Transl Oncol 20: 1353-1357, 2018.

5. Baur M, Kienzer HR, Rath T and Dittrich C: Extravasation of oxaliplatin (Eloxatin((R)))-clinical course. Onkologi 23: 468-471, 2000.

6. Pérez Fidalgo JA, García Fabregat L, Cervantes A, Margulies A, Vidall $\mathrm{C}$ and Roila F; ESMO Guidelines Working Group: Management of chemotherapy extravasation: ESMO-EONS clinical practice guidelines. Ann Oncol 23 (Suppl 7): vii167-vii173, 2012.

7. Pericay C, López A, Soler JR, Bonfill T, Dotor E and Saigí E: Extravasation of oxaliplatin: An infrequent and irritant toxicity. Clin Transl Oncol 11: 114-116, 2009.

8. Wickham R, Engelking C, Sauerland C and Corbi D: Vesicant extravasation part II: Evidence-based management and continuing controversies. Oncol Nurs Forum 33: 1143-1150, 2006.

9. Kreidieh FY, Moukadem HA and El Saghir NS: Overview, prevention and management of chemotherapy extravasation. World J Clin Oncol 7: 87-97, 2016.

10. Narducci F, Jean-Laurent M, Boulanger L, El Bédoui S, Mallet Y, Houpeau JL, Hamdani A, Penel N and Fournier C: Totally implantable venous access port systems and risk factors for complications: A one-year prospective study in a cancer centre. Eur J Surg Oncol 37: 913-918, 2011.

11. Masters B, Hickish T and Cidon EU: A midline for oxaliplatin infusion: The myth of safety devices. BMJ Case Rep 2014: bcr2014204360, 2014.

12. Boesen $C$ and Nielsen SE: Reversible tissue damage after paravasal infusion of oxaliplatin. Ugeskr Laeger 177: V07140390, 2015 (In Danish).

13. Leon-Ferre RA, Abu Hejleh TB and Halfdanarson TR: Extravasation of oxaliplatin into the mediastinum: A case report and review of the literature. Clin Adv Hematol Oncol 10: 546-548, 2012.

14. Haslik W, Hacker S, Felberbauer FX, Thallinger C, Bartsch R, Kornauth C, Deutschmann C and Mader RM: Port-a-Cath extravasation of vesicant cytotoxics: Surgical options for a rare complication of cancer chemotherapy. Eur J Surg Oncol 41: 378-385, 2015.

15. Azaïs H, Bresson L, Bassil A, Katdare N, Merlot B, Houpeau JL, El Bedoui S, Meurant JP, Tresch E and Narducci F: Chemotherapy drug extravasation in totally implantable venous access port systems: How effective is early surgical lavage? J Vasc Access 16: 31-37, 2015. 
16. Makrilia N, Syrigou E, Kaklamanos I, Manolopoulos L and Saif MW: Hypersensitivity reactions associated with platinum antineoplastic agents: A systematic review. Met Based Drugs 2010: 207084, 2010.

17. Sorich J, Taubes B, Wagner A and Hochster H: Oxaliplatin: Practical guidelines for administration. Clin $\mathrm{J}$ Oncol Nurs 8 251-256, 2004.

18. Markman M: Toxicities of the platinum antineoplastic agents. Expert Opin Drug Saf 2: 597-607, 2003.

19. de Lemos ML and Walisser S: Management of extravasation of oxaliplatin. J Oncol Pharm Pract 11: 159-162, 2005.
20. Kretzschmar A, Pink D, Thuss-Patience P, Dörken B, Reichert P and Eckert R: Extravasations of oxaliplatin. J Clin Oncol 21: 4068-4069, 2003.

21. Foo KF, Michael M, Toner G and Zalcberg J: A case report of oxaliplatin extravasation. Ann Oncol 14: 961-962, 2003.

22. Conde-Estévez D and Mateu-de Antonio J: Update in the management of extravasations of cytocytostatic agent. Farm Hosp 36: 34-42, 2012 (In Spanish). 